\title{
Multi-objective Optimization Approach to Malfatti's Problem
}

\author{
Rentsen Enkhbat ${ }^{1}$ and Gompil Battur ${ }^{2}$ \\ 1 Institute of Mathematics and Digital Technology, Academy of Sciences of Mongolia, \\ Ulaanbaatar, Mongolia \\ E-mail: renkhbat46@yahoo.com \\ 2 Department of Applied Mathematics, National university of Mongolia, Ulaanbaatar, \\ Mongolia \\ E-mail: battur@seas.num.edu.com
}

\begin{abstract}
In this work, we consider the multi-objective optimization problem based on the circle packing problem, particularly, extended Malfatti's problem (Enkhbat, 2020) with $k$ disks. Malfatti's problem was examined for the first time from a view point of global optimization theory and algorithm in (Enkhbat, 2016). Also, a game theory approach has been applied to Malfatti's problem in (Enkhbat and Battur, 2021). In this paper, we apply the the multi-objective optimization approach to the problem. Using the weighted sum method, we reduce this problem to optimization problem with nonconvex constraints. For solving numerically the weighted sum optimization problem, we apply KKT conditions and find Pareto stationary points. Also, we estimate upper bounds of the global value of the objective function by Lagrange duality. Numerical results are provided.
\end{abstract}

Keywords: circle packing problem, triangle set, $k$ disks, multi-objective optimization problem, upper bound.

\section{Introduction}

In recent years, circle packing problem have attracted many researchers because of its applications such as circular cutting problems, communication networks, facility location and dashboard layout. For instance, we refer to (Nguyen and Strodiot, 1992) for the practical application of the design centering problem in the diamond industry, to (Vidigal and Director, 1982) for the quality control problem, to (Fraser and George, 1994) for container loading problem for pulp industries which reduces to the circle packing problem, to (Dowsland, 1991) for the problem of the cylindrical units into a rectangular container.

A well-known Italian mathematician Malfatti (Malfatti, 1803) proposed the problem of how to pack three non-overlapping disks of maximum total area in a given triangle. It is shown (Enkhbat, 2020) that this problem is a particular case of the circle packing problem. Now it is well known that Malfatti's solution is not the optimal one. A number of works attempted to solve Malfatti's problem (Enkhbat, 2016; Enkhbat and Barkova, 2019; Enkhbat, 2020; Enkhbat and Battur, 2021; Andreatta et al., 2011; Zalgaller and Los, 1994; Los, 1988; Lob and Richmond, 1930; Malfatti, 1803). Algebraic as well as geometric approaches have been applied to find the optimal solutions to the problem. Zalgaller and Los (1994) showed that the greedy arrangement is the most optimal one for the problem solution. They applied the so-called rigid systems and found the best solution to the problem which was formulated on the basis of trigonometric equations and inequalities. In (Enkhbat, https://doi.org/10.21638/11701/spbu31.2021.07 
2016), Malfatti's problem was considered as a global optimization problem. Generalized Nash equilibrium problem based on Malfatti's problem has been formulated in (Enkhbat and Battur, 2021). In this paper, we examine the extended Malfatti's problem (Enkhbat, 2020) with $k$ disks from a viewpoint of multi-objective optimization problem. The paper is organized as follows. In section 2 , we recall the circle packing problem as the convex maximization problem. In section 3 , first, we formulate multi-objective optimization problem using the extended Malfatti's problem. In section 4, we consider the KKT conditions and Lagrange dual problem. In section 5 , we provide with numerical results.

\section{Formulation of Sphere Packing Problem}

Enkhbat $(2016 ; 2020)$ formulated statements of the problem and optimality conditions for sphere packing as well as Malfatti's problem (Malfatti, 1803). Now we recall the sphere packing problem formulation with $k$ disks briefly. Let $D$ be a polyhedral set given by the following linear inequalities.

$$
D=\left\{x \in \mathbb{R}^{n} \mid\left\langle a^{i}, x\right\rangle \leq b_{i}, i=\overline{1, m}\right\}, a^{i} \in \mathbb{R}^{n}, b_{i} \in \mathbb{R} .
$$

Assume that $D$ is compact and int $\neq \neq \emptyset$. Then the sphere packing problem has the form (Enkhbat, 2020)

$$
\max _{(u, r)} V=\frac{\pi^{n / 2}}{\Gamma\left(\frac{n}{2}+1\right)} \sum_{i=1}^{k} r_{i}^{n}
$$

subject to:

$$
\begin{gathered}
\left\langle a^{i}, u^{j}\right\rangle+r_{j}\left\|a^{i}\right\|-b_{i} \leq 0, i=\overline{1, m}, j=\overline{1, k} \\
\left(r_{i}+r_{j}\right)^{2}-\left\|u^{i}-u^{j}\right\|^{2} \leq 0, i, j=\overline{1, k}, i<j \\
-r_{j} \leq 0, j=1, \ldots, k
\end{gathered}
$$

where $\Gamma(\cdot)$ is the gamma function. The function $V$, which is convex as a sum of convex functions $r_{i}^{n}$ defined on the positive orthant of $\mathbb{R}$, denotes the total volume of all spheres inscribed in $D . u^{i}$ are centers of disks, $i=1, \ldots, k$. Conditions (2) describe that all spheres are in $D$ while conditions (3) secure non-overlapping case of balls. Denote by $S P(n, m, k)$ problem (1)-(4), where $n, m$ and $k$ are its parameters. It is clear that $S P(2,3,3)$ is Malfatti's problem.

If we set $r_{j}=r, j=1,2, \ldots, k$, then problem (1)-(4) reduces to the classical packing problem of inscribing $k$ equal spheres into $D$ with maximum volume:

$$
\max _{(u, r)} V=\frac{\pi^{n / 2}}{\Gamma\left(\frac{n}{2}+1\right)} k r^{n}
$$

subject to:

$$
\begin{gathered}
\left\langle a^{i}, u^{j}\right\rangle+r\left\|a^{i}\right\| \leq b_{i}, i=\overline{1, m}, j=\overline{1, k} \\
\left\|u^{i}-u^{j}\right\|^{2} \geq 4 r^{2}, i, j=\overline{1, k}, i<j \\
-r \leq 0 .
\end{gathered}
$$




\section{Multi-objective optimization formulation of Malfatti's problem}

We consider the problem $S P(n, m, k)$ which is the extension of Malfatti's problem with $k$ disks. Let us now consider this problem from a point of view of game theory. Suppose $k$ players are involved in the game. Assume that $k$ players who correspond to each disk have to maximize their area simultaneously. One can denote by $y:=(u, r) \in \mathbb{R}^{n+1}$. In this case, we introduce $l:=\frac{k(k-1)}{2}$ and $g(y)=$ $\left(g_{1}(y), \ldots, g_{m \cdot k}(y)\right)^{T}, h(y)=\left(h_{1}(y), \ldots, h_{l}(y)\right)^{T}$ and $s(y)=\left(s_{1}(y), \ldots, s_{k}(y)\right)^{T}$ as the left side vector-functions of the constraints (2), (3) and (4) respectively. In other words,

$$
\left\{\begin{array}{c}
g_{1}(y)=\left\langle a^{i}, u^{i}\right\rangle+r_{j}\left\|a^{i}\right\|-b_{i} \\
\cdots \\
g_{m \cdot k}(y)=\left\langle a^{m}, u^{k}\right\rangle+r_{k}\left\|a^{m}\right\|-b_{m} \\
h_{1}(y)=\left(r_{1}+r_{2}\right)^{2}-\left\|u^{1}-u^{2}\right\|, \\
\cdots \cdots \\
h_{l}(y)=\left(r_{k-1}+r_{k}\right)^{2}-\left\|u^{k-1}-u^{k}\right\|, \\
s_{j}(y)=-r_{j}, j=1, \ldots, k .
\end{array}\right.
$$

Denote by $S$ the feasible set of problem (1)-(4)

$$
S=\left\{y \in \mathbb{R}^{n+1} \mid g(y) \underset{\mathbb{R}^{m \cdot k}}{\leq} 0, h(y) \underset{\mathbb{R}^{l}}{\leq} 0, s(y) \underset{\mathbb{R}^{k}}{\leq} 0\right\} .
$$

Let us consider multi-objective optimization problem 23 based on Malfatti's problem in the following:

$$
\left\{\begin{array}{c}
\max _{y \in S} f_{1}(y)=\pi r_{1}^{2}, \\
\ldots \ldots \\
\max _{y \in S} f_{k}(y)=\pi r_{k}^{2} .
\end{array}\right.
$$

Denote the problem (9) by $\operatorname{MOSP}(n, m, k)$.

Definition 1. $\bar{y} \in S$ is called a Pareto optimal point of $\operatorname{MOSP}(n, m, k)$ if there is no $y \in S$ with

$$
f_{i}(y) \geq f_{i}(\bar{y}) \text { for all } i \in\{1, \ldots, k\}
$$

and $f(y) \neq f(\bar{y}), \quad f=\left(f_{1}, \ldots, f_{k}\right)$.

The Pareto optimal concept is the main optimality notion used in multi-objective optimization. The main approach for determination of Pareto optimal point is the weighted sum approach. Therefore, one can introduce appropriate weights $\alpha_{1}>$ $0, \ldots, \alpha_{k}>0, \sum_{i=1}^{k} \alpha_{i}=1$, so we can formulate the corresponding scalarized optimization problem for problem (9):

$$
\max _{y \in S} F(y)=\sum_{i=1}^{k} \alpha_{i} f_{i}(y)
$$

Problem (10) can be also considered as the convex maximization problem over nonconvex constraint set. A relationship between Pareto optimal point and solution of the scalarized problem is given by the following well-known result (Ehrgott, 2005). 
Proposition 1. A solution $y^{\star}$ to problem (10) is a Pareto optimal point of problem $\operatorname{MOSP}(n, m, k)$.

Proof. Let $y^{\star}$ be a solution to problem (10) but is not a Pareto optimal point of problem (9). This means that there exist a point $\tilde{y} \in S$ such that $f_{i}(\tilde{y}) \geq f_{i}\left(y^{\star}\right)$ for all $i \in\{1, \ldots, k\}$ with $f_{k}(\tilde{y})>f_{k}\left(y^{\star}\right), \exists j \in\{1, \ldots, k\}$.

$$
F\left(y^{\star}\right)=\sum_{i=1}^{k} \alpha_{i} f_{i}\left(y^{\star}\right) \leq \sum_{i=1}^{k} \alpha_{i} f_{i}(\tilde{y})<\sum_{i \neq j}^{k} \alpha_{i} f_{i}(\tilde{y})+f_{j}(\tilde{y})=F(\tilde{y})
$$

This contradicts that $y^{\star}$ is a solution to problem (10).

\section{KKT condition and Lagrange dual problem}

In this section we consider the KKT conditions and formulate well-known Lagrange dual problem (Bertsekas, 2016) for problem (10). One purpose of Lagrange duality is to find an upper bound for optimization problem. Formally, the Lagrangian function associated with the optimization problem (10) is defined as

$$
L(y, \lambda, \mu, \beta)=F(y)-\left(\sum_{i=1}^{m \cdot k} \lambda_{i} g_{i}(y)+\sum_{i=1}^{l} \mu_{i} h_{i}(y)+\sum_{i=1}^{k} \beta_{i} s_{i}(y)\right) .
$$

The dual variables $\lambda \in \mathbb{R}_{+}^{m \cdot k}, \mu \in \mathbb{R}_{+}^{l}$ and $\beta \in \mathbb{R}_{+}^{k}$ are also referred to as the Lagrange multipliers associated with the inequality constraints. Now we write the KKT conditions for the problem (10) as the following:

$$
\left\{\begin{array}{l}
\nabla F\left(y^{*}\right)-\left(\sum_{i=1}^{m \cdot k} \lambda_{i}^{*} \nabla g_{i}\left(y^{*}\right)+\sum_{i=1}^{l} \mu_{i}^{*} \nabla h_{i}\left(y^{*}\right)+\sum_{i=1}^{k} \beta_{i}^{*} \nabla s_{i}\left(y^{*}\right)\right)=0, \\
\lambda_{i}^{*} g_{i}\left(y^{*}\right)=0, \lambda_{i}^{*} \geq 0, g_{i}\left(y^{*}\right) \leq 0, i=1, \ldots, m \cdot k, \\
\mu_{i}^{*} h_{i}\left(y^{*}\right)=0, \mu_{i}^{*} \geq 0, h_{i}\left(y^{*}\right) \leq 0, i=1, \ldots, l, \\
\beta_{i}^{*} s_{i}\left(y^{*}\right)=0, \beta_{i}^{*} \geq 0, \quad s_{i}\left(y^{*}\right) \leq 0, i=1, \ldots, k .
\end{array}\right.
$$

Denote by $p^{*}$ the optimal value of the primal problem (10) which exists due to compactness of set $S$ :

$$
p^{*}=\max _{y \in S}(F(y)) .
$$

Define the Lagrange dual function $\Phi: \mathbb{R}^{m \cdot k} \times \mathbb{R}^{l} \times \mathbb{R}^{k} \rightarrow \mathbb{R}$ over the dual variables as

$$
\Phi(\lambda, \mu, \beta)=\sup _{y \in \mathbb{R}^{n+1}} L(y, \lambda, \mu, \beta),
$$

and the associated dual optimization problem over the dual variables as

$$
\begin{array}{r}
\inf \Phi(\lambda, \mu, \beta) \\
\text { subject to: } \lambda \underset{\mathbb{R}^{m \cdot k}}{\geq} 0, \mu \underset{\mathbb{R}^{l}}{\geq} 0, \beta \underset{\mathbb{R}^{k}}{\geq} 0 .
\end{array}
$$

It is well known that this dual problem is always a convex optimization problem, even if the primal problem is not convex (note that the dual function is a pointwise supremum of affine functions of $(\lambda, \mu, \beta)$, and therefore concave). Let $C_{S}$ denote the constraint set of this dual problem. Any point $(\lambda, \mu, \beta) \in C_{S}$ is said to be a dual feasible. Denote by $d^{*}$ the optimal value of the dual problem:

$$
d^{*}=\inf _{(\lambda, \mu, \beta) \in C_{S}} \Phi(\lambda, \mu, \beta) .
$$


Proposition 2. Let $y$ and $(\lambda, \mu, \beta)$ be feasible in $(10)$ and $\left(D_{L}\right)$, respectively. For given $\alpha_{1}, \ldots, \alpha_{k} \geq 0$,

$$
p(y) \leq d(\lambda, \mu, \beta) .
$$

If $p^{*}(y)=d^{*}(\lambda, \mu, \beta)$, then the pair $(y,(\lambda, \mu, \beta))$ is optimal in its respective problem. Proof. Proof follows from the duality theory (Bertsekas, 2016).

Thus the optimal value of the dual problem gives an upper bound for the optimal value of the primal problem. Denote by

$$
\rho(\lambda, \mu, \beta, y)=d(\lambda, \mu, \beta)-p(y)
$$

the difference of $d(\lambda, \mu, \beta)$ and $p(y)$ functions. Obviously, for $(\lambda, \mu, \beta) \in C_{S}, y \in S$, we have $\rho(\lambda, \mu, \beta, y) \geq 0$.

\section{Computational Results}

In this section, we consider the problem $S P(2,3,3)$ in two cases. The scalarized problem (10) is a nonconvex optimization problem which is NP hard problem as the convex maximization problem. Nonconvex optimization problem have multiple local optimal points. Hence, it may be reasonable to try to find a stationary solution. Here, a stationary point means a point that satisfies the first-order optimality condition (KKT condition). We use Matlab's lsqnonlin() function for solving the KKT condition (12). The numerical results were implemented on an Intel(R) Core(TM) i7, CPU 3.20GHz processor workstation under Matlab R2017b. We run the algorithm in Matlab: 'trust-region-reflective' and 'exitflag $=1$ '. We considered the following steps in the computational method.

1. For each fixed different weights, we solve the KKT condition of the problem (10) and find any stationary point $y^{*}$.

2. For fixed $y^{*}$, we find a solution of the problem $\left(D_{L}\right)$ which is linear programming problem.

3. If $\left(y^{*}, \lambda^{*}, \mu^{*}, \beta^{*}\right)$ is satisfies the following condition:

$$
\left|\rho\left(\lambda^{*}, \mu^{*}, \beta^{*}, y^{*}\right)\right| \leq \epsilon, \quad \epsilon=10^{-5}
$$

then, go to next step.

4. Compare total areas for each different weights. We choose a Pareto stationary point which corresponds to the maximum total area. Go to step 1. Update weights again.

Example 1 For a test purpose, the triangle with vertices $A(2,2), B(4,19)$ and $C(24,8)$ has been considered. The triangle set is given by the following system of linear inequalities:

$$
\begin{gathered}
-17 y_{1}+2 y_{2} \leq-30, \\
11 y_{1}+20 y_{2} \leq 424, \\
6 y_{1}-22 y_{2} \leq-32 .
\end{gathered}
$$

For problem $\operatorname{MOSP}(2,3,3)$, the scalarized problem (10) is formulated as:

$$
\max \pi\left(\alpha_{1} y_{3}^{2}+\alpha_{2} y_{6}^{2}+\alpha_{3} y_{9}^{2}\right)
$$




$$
\begin{gathered}
g_{1}(y)=-17 y_{1}+2 y_{2}+17.1172 y_{3}+30 \leq 0, \\
g_{2}(y)=11 y_{1}+20 y_{2}+22.8254 y_{3}-424 \leq 0, \\
g_{3}(y)=6 y_{1}-22 y_{2}+22.8035 y_{3}+32 \leq 0, \\
g_{4}(y)=-17 y_{4}+2 y_{5}+17.1172 y_{6}+30 \leq 0, \\
g_{5}(y)=11 y_{4}+20 y_{5}+22.8254 y_{6}-424 \leq 0, \\
g_{6}(y)=y_{4}-22 y_{5}+22.8035 y_{6}+32 \leq 0, \\
g_{7}(y)=-17 y_{7}+2 y_{8}+17.1172 y_{9}+30 \leq 0, \\
g_{8}(y)=11 y_{7}+20 y_{8}+22.8254 y_{9}-424 \leq 0, \\
g_{9}(y)=6 y_{7}-22 y_{8}+22.8035 y_{9}+32 \leq 0, \\
h_{1}(y)=\left(y_{3}+y_{6}\right)^{2}-\left(y_{4}-y_{1}\right)^{2}-\left(y_{5}-y_{2}\right)^{2} \leq 0, \\
h_{2}(y)=\left(y_{3}+y_{9}\right)^{2}-\left(y_{7}-y_{1}\right)^{2}-\left(y_{8}-y_{2}\right)^{2} \leq 0, \\
h_{3}(y)=\left(y_{6}+y_{9}\right)^{2}-\left(y_{7}-y_{4}\right)^{2}-\left(y_{8}-y_{5}\right)^{2} \leq 0, \\
s_{1}(y)=-y_{3} \leq 0, s_{2}(y)=-y_{6} \leq 0, s_{3}(y)=-y_{9} \leq 0 .
\end{gathered}
$$

The results are given as the following:

1. For $\alpha_{1}=0.3, \alpha_{2}=0.3, \alpha_{3}=0.4$,

$y_{1}^{*}=(13.1056,9.2948,4.1157,6.8259,13.5107,3.4479,6.0172,6.6659,3.4445)$,

$\left(\lambda^{*}, \mu^{*}, \beta^{*}\right)=(0,0.03,0.03,0,0,0,0,0,0.03,0,0.03,0,0,0,0)$,

Total area $S_{1}=127.8364$.

2. For $\alpha_{1}=0.4, \alpha_{2}=0.3, \alpha_{3}=0.3$,

$y_{2}^{*}=(8.7285,9.8150,5.7693,3.9008,4.2078,1.6299,17.0607,8.8247,2.6215)$,

$\left(\lambda^{*}, \mu^{*}, \beta^{*}\right)=(0,0.012,0.012,0.012,0,0,0,0,0.012,0.012,0,0,0,0,0)$,

Total area $S_{2}=134.5031$.

3. For $\alpha_{1}=0.5, \alpha_{2}=0.1, \alpha_{3}=0.4$,

$y_{3}^{*}=(5.3397,16.3977,1.6345,4.1585,14.3885,0.6962,8.7285,9.8150,5.7693)$,

$\left(\lambda^{*}, \mu^{*}, \beta^{*}\right)=(0,0,0,0,0,0,0,0.022,0.022,0.022,0.022,0.022,0,0,0)$,

Total area $S_{3}=114.4831$.

From the numerical results, Case 2 gives the best stationary Pareto point corresponding to the maximum area.

Example 2 Now consider the triangle with vertices $A(0,0), B(3,4)$ and $C(8,6)$. Then problem (10) is formulated in the following:

$$
\begin{gathered}
\max f=\pi\left(\alpha_{1} y_{3}^{2}+\alpha_{2} y_{6}^{2}+\alpha_{3} y_{9}^{2}\right), \\
g_{1}(y)=-4 y_{1}+3 y_{2}+5 y_{3} \leq 0, \\
g_{2}(y)=6 y_{1}-8 y_{2}+10 y_{3} \leq 0, \\
g_{3}(y)=-2 y_{1}+5 y_{2}+\sqrt{29} y_{3}-14 \leq 0, \\
g_{4}(y)=-4 y_{4}+3 y_{5}+5 y_{6} \leq 0, \\
g_{5}(y)=6 y_{4}-8 y_{5}+10 y_{6} \leq 0,
\end{gathered}
$$




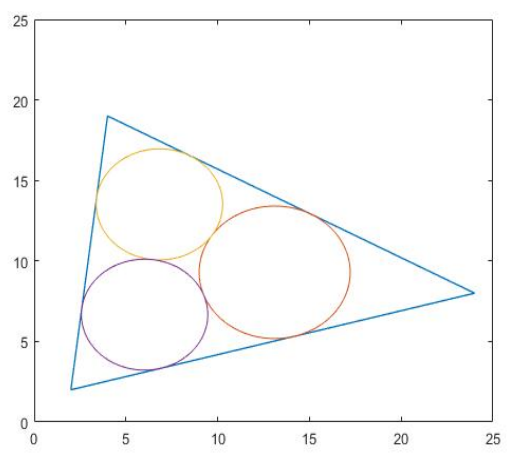

Fig. 1. Case 2, $\alpha_{1}=0.4, \alpha_{2}=0.3, \alpha_{3}=0.3$

$$
\begin{gathered}
g_{6}(y)=-2 y_{4}+5 y_{5}+\sqrt{29} y_{6}-14 \leq 0, \\
g_{7}(y)=-4 y_{7}+3 y_{8}+5 y_{9} \leq 0, \\
g_{8}(y)=6 y_{7}-8 y_{8}+10 y_{9} \leq 0, \\
g_{9}(y)=-2 y_{7}+5 y_{8}+\sqrt{29} y_{9}-14 \leq 0, \\
h_{1}(y)=\left(y_{3}+y_{6}\right)^{2}-\left(y_{4}-y_{1}\right)^{2}-\left(y_{5}-y_{2}\right)^{2} \leq 0, \\
h_{2}(y)=\left(y_{3}+y_{9}\right)^{2}-\left(y_{7}-y_{1}\right)^{2}-\left(y_{8}-y_{2}\right)^{2} \leq 0, \\
h_{3}(y)=\left(y_{6}+y_{9}\right)^{2}-\left(y_{7}-y_{4}\right)^{2}-\left(y_{8}-y_{5}\right)^{2} \leq 0, \\
s_{1}(y)=-y_{3} \leq 0, s_{2}(y)=-y_{6} \leq 0, s_{3}(y)=-y_{9} \leq 0 .
\end{gathered}
$$

Computational results for the above example are given as the following.

1. For $\alpha_{1}=0.5, \alpha_{2}=0.1, \alpha_{3}=0.4$, $y_{1}^{*}=(3.4339,3.4339,0.6868,2.5830,2.5830,0.5166,4.4925,4.0288,0.5276)$, $\left(\lambda^{*}, \mu^{*}, \beta^{*}\right)=(0,0,0,0.00012,0,0,0,0,0,0.00012,0.00012,0,0,0,0)$,

Total area $S_{1}=3.1948$.

2. For $\alpha_{1}=0.5, \alpha_{2}=0.3, \alpha_{3}=0.2$, $y_{2}^{*}=(4.7580,4.1776,0.4595,2.4100,2.4096,0.4298,3.3006,3.2845,0.5857)$, $\left(\lambda^{*}, \mu^{*}, \beta^{*}\right)=(0,0,0,0.0002,0,0,0,0,0,0,0,0,0,0,0)$,

Total area $S_{2}=2.3214$.

Case 1 corresponds to the best stationary Pareto point.

\section{Conclusion}

In this paper, we have examined a multi-objective optimization problem based on the sphere packing problem as well as Malfatti's problem. The problem was reduced to a scalarized optimization problem with nonconvex constraints. Numerical experiments allow us to find some stationary Pareto solutions for given weights. We obtain the best stationary Pareto point comparing the total areas for different weights of the scalarized problem. Numerical results are given. 


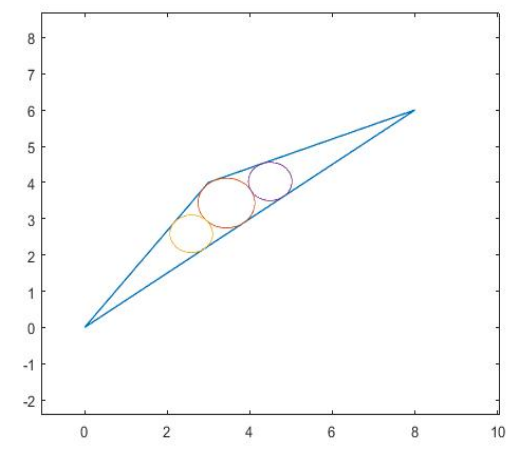

Fig. 2. Case $1, \alpha_{1}=0.5, \alpha_{2}=0.1, \alpha_{3}=0.4$

\section{References}

Nguyen, V. H. and J. J. Strodiot (1992). Computing a Global Optimal Solution to a Design Centering Problem. Mathematical Programming, 53, 111-123.

Vidigal, L. M. and S.W. Director (1982). A Design Centering Algorithm for Nonconvex Regions of Acceptability. IEEE Transactions on Computer-Aided-Design of Integrated Circuits and Systems, CAD-1 pp. 13-24.

Fraser, H. J. and J. A. George (1994). Integrated container loading software for pulp and paper industry. European Journal of Operational Research, 77, 466-474.

Dowsland, K. A. (1991). Optimising the palletisation of cylinders in cases. OR Spectrum 13, 204-212.

Enkhbat, R. (1996). An algorithm for maximizing a convey function over a simple set. Journal of Global Optimization, 8, 379-391.

Enkhbat, R. (2016). Global optimization approach to Malfatti's problem. Journal of Global Optimization, 65, 33-39.

Enkhbat, R. and M. Barkova (2019). Global search method for solving Malfatti's four-circle problem. The Bulletin of Irkutsk State University. Series Mathematics, 15, 38-49.

Enkhbat, R. (2020). Convex Maximization Formulation of General Sphere Packing Problem. The Bulletin of Irkutsk State University. Series Mathematics, 31, 142-149.

Enkhbat, R., Battur, G. (2021). Generalized Nash equilibrium problem based on Malfatti's problem. Numerical Algebra, Control and Optimization, 11(2), 209-220.

Andreatta, M., Bezdek, A., Boronski, J. P.(2011). The Problem of Malfatti: Two Centuries of Debate. The Mathematical Intelligencer, 33(1), 72-76.

Nefedov, V. N. (1987). Finding the Global Maximum of a Function of Several Variables on a Set Given by Inequality Constraints. Journal of Numerical Mathematics and Mathematical Physics, 27(1), 35-51.

Strekalovsky, A.S. (1987). On the global extrema problem. Soviet Math. Doklad, 292(5), 1062-1066.

Zalgaller, V. A. (1991). An inequality for acute triangles. Ukr. Geom. Sb., 34, 10-25.

Zalgaller, V. A. and G. A. Los (1994). The solution of Malfatti's problem. Journal of Mathematical Sciences, 72(4), 3163-3177.

Los, G. A. (1988). Malfatti's Optimization Problem [in Russian], Dep. Ukr. NIINTI July $5,1988$.

Saaty, T. (1973). Integer Optimization Methods and Related Extremal Problems [Russian translation], Moscow.

Gabai, H. and E. Liban (1967). On Goldberg's inequality associated with the Malfatti problem. Math. Mag., 41(5), 251-252. 
Goldberg, M. (1967). On the original Malfatti problem. Math. Mag., 40(5), 241-247.

Lob, H. and H. W. Richmond (1930). On the solutions of the Malfatti problem for a triangle. Proc. London Math. Soc., 2(30), 287-301.

Malfatti, C. (1803). Memoria sopra una problema stereotomico. Memoria di Matematica e di Fisica della Societa ttaliana della Scienze, 10(1), 235-244.

Boyd, S. and L. Vandenberghe (2004). Convex Optimization, Cambridge University Press.

Bertsekas, D. P. (2016). Nonlinear programming.

Ehrgott, M. (2005). Multicriteria Optimization, Springer Berlin-Heidelberg. 